\title{
A neural approach to drugs monitoring for personalized medicine
}

\author{
Benjamin Staar ${ }^{1 \dagger}$, Marius Schirmer ${ }^{2 \dagger}$, Camilla Bai-Rossi ${ }^{3 \dagger}$, Giovanni De Micheli ${ }^{3}$, Sandro Carrara ${ }^{3}$ and Elisabetta Chicca $^{2}$ \\ ${ }^{1}$ Jacobs University \\ Bremen, Germany \\ ${ }^{2}$ Faculty of Technology and Cognitive Interaction Technology - Center of Excellence, Bielefeld University \\ Bielefeld, Germany \\ ${ }^{3}$ Integrated System Labratory, EPFL - École Polytechnique Fédérale de Lausanne, Switzerland \\ Email: mschirmer@uni-bielefeld.de
}

\begin{abstract}
The development of fast and mobile drug detection is an important aspect of personalized medicine. It enables the quick assessment of inter-individual differences in drug metabolism and corresponding adjustments of the dose. Recent developments of amperometric biosensors using cytochrome $\mathbf{P 4 5 0}$ (CYP) show great promise, by lowering the detection limit to physiological range for several drugs via the usage of Multi Walled Carbon Nanotubes (MWCNT). The next challenge is to develop algorithms for processing the resulting sensor data compatible with low-power hardware, which would allow the development of portable battery-powered devices. In this work we pursue a novel approach to this problem. Here we provide a proof of principle by demonstrating how sensor data could be analyzed using a conventional multi-layer perceptron network with error-backpropagation.
\end{abstract}

\section{INTRODUCTION}

Ideally medical substances would affect each patient equally. However, this is rarely the case as subjects show inter-individual differences in metabolizing different types of drugs. This constitutes an important problem in personalized medicine that opens to new challenges in bioelectronics [1]. A possible solution is to analyze the patients metabolization rate before drug administration. However, this process is often time consuming and requires expensive and bulky equipment which hinders wide-spread application. Consequently there is a requirement for fast, cheap and mobile solutions. A possible candidate for the development of such mobile devices is amperometric biosensing employing cytochrome P450 (CYP), an enzyme involved in the metabolism of a wide variety of drugs [2]. Recent efforts in lowering the detection limit to physiological ranges via the usage of Multi Walled Carbon Nanotubes (MWCNT) [3] have shown to be successful for several drugs [4]. However there are still challenges on the way towards broader or even commercial applications of these sensors. One crucial problem is the low specificity of the CYP probes against several different drug compounds. [5]. A possible solution is to acquire calibration curves with interdependence among different drugs on the same biosensor [3]. Another one is to use the matrix approach on arrays of biosensors [6]. However, all these approaches are linear while the problem of drug-drug interaction on the single CYP

\footnotetext{
${ }^{\dagger}$ Authors equally contributed to this work.
}

probe is clearly not [7]. A neural network approach with backpropagation, on the other hand, is capable of approximating non-linear functions. A further challenge is the development of algorithms for automatic analysis of the sensory data compatible with low power implementation and therefore suitable for the design of portable battery-powered devices. A first step is automating data preprocessing and demonstrating how neural networks can be used to directly predict drug concentration(s) from this preprocessed data. Given the limited data available from the sensors, a major limitation for a neural network approach is the training, which normally relies on a big data set. In order to overcome this problem, we introduce a method to generate training data by interpolating between real measurements from a set of calibration data. We also provide a proof of concept for the application of this method to multipledrug measurements.

\section{METHODS}

\section{A. Electrochemical measurements}

Screen-printed electrodes were functionalized with Multi Walled Carbon Nanotubess (MWCNTs) and microsomal cytochrome P450 (CYP) as described in [3], [7]. Amperometric biosensors based on the CYP enzyme family are widely investigated in research, because CYP is an enzyme primarily involved in the metabolism of drugs and steroids [8]. In the absence of the CYP substrate, CYP can react with oxygen when a redox potential is applied to the electrode. This reaction can be quantified by analyzing the reduction peaks in cyclic voltammetry measurements. The faradaic current associated with the reduction peak is dependent on the quantity of CYP immobilized onto the electrode, and the peak potential is influenced by the immobilization technique. When the drug (substrate) is added to a buffer solution in presence of oxygen, a further increase in the CYP reduction current is observed. Several CYP isoforms were used for the specific detection of the selected drugs: the CYP2C9 for Naproxen and Flurbiprofen, CYP1A2 for Ftorafur and Etoposide, and CYP3A4 for Ifosfamide and Etoposide [3], [7].

\section{B. Data preprocessing}

In order to enable fully automated data processing, we developed a standardized way for the preprocessing of voltammetric data shown in Fig. 1: First the reduction part, i.e. 


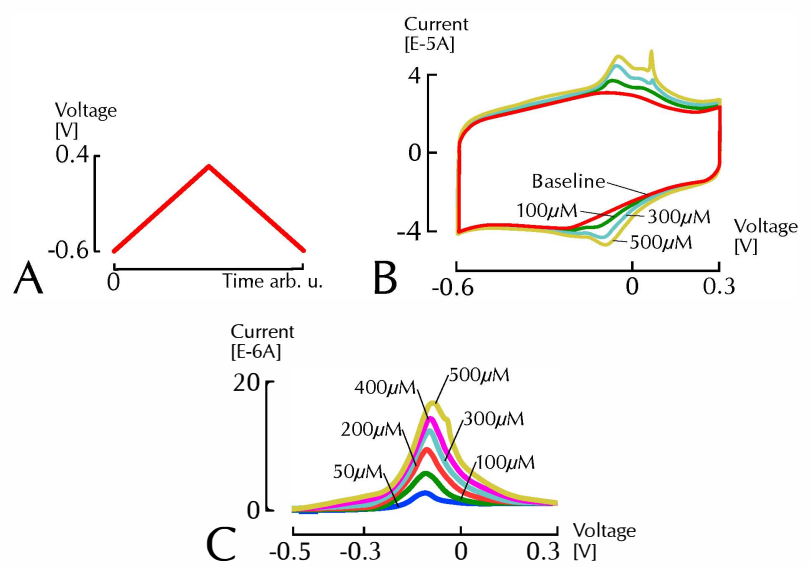

Fig. 1: Example of how to obtain the data. A: applied voltage ramping, B: resulting current loop, $\mathrm{C}$ : preprocessed curves with baseline subtraction.

the currents acquired from the negative voltage sweep, were isolated. We then subtracted the baseline current which was acquired by measurements in Phosphate-Buffered Saline (PBS) without the presence of any drug. The resulting traces were normalized so that peaks resulting from drug measurements were positive, i.e. if the peaks were negative after background current subtraction they were multiplied by -1 . The approximate voltage ranges for each drug peaks were known from previous research (Naproxen and Flurbiprofen [7]; Etoposide, Ftorafur, Ifosfamid and Cyclophosphamide [3])

\section{Interpolating of data}

Our aim is to train a neural network to predict single or cocktail drug concentration from preprocessed sensor data. The goal of this approach is to avoid computationally expensive data processing and therefore facilitate the development of portable drug detection devices. However training and testing a neural network to predict drug concentration requires sufficiently large sets of training and testing data. Given the large extent of sensor-to-sensor variability, the training set has to be acquired for each sensor individually. Due to time-consuming biochemical experiments, the size of the calibration data set is not large enough to split it in reasonable sized training and testing data sets. Therefore, we developed a simple algorithm to interpolate between measurements from a small calibration data set, allowing the creation of arbitrarily large simulated data sets for training and testing. Under ideal conditions a measured drug would give a single peak in the reduction part of the voltammogram of approximately Gaussian shape, with a linearly dependent amplitude of the drug's concentration [1] [9]. However, since we are dealing with a complex system consisting of the CYP plus microsome, MWCNT, PBS and one or multiple drugs, we often get much more complex responses. We accounted for this problem by fitting with a sum of $N$ Gaussians the response in current $I_{k}$ to the input voltage $V$ in presence of a single drug

$$
I_{k}(V)=\sum_{i=1}^{N} a_{i} \mathrm{e}^{\frac{-\left(V-\boldsymbol{p}_{i}\right)^{2}}{2 \sigma_{i}^{2}}},
$$

with the Gaussian components amplitude $a_{i} \geq 0$, position $p_{i}$ and width $\sigma_{i}$. Given suitable sensor quality, at least one of these Gaussian components will show a linear concentration

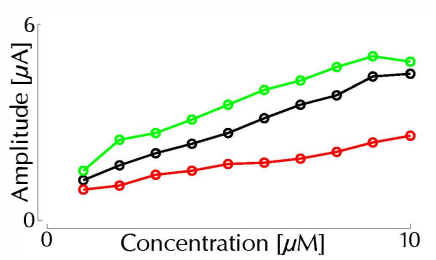

(a) Measured data from Ftorafur

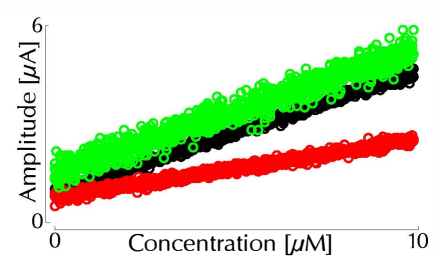

(b) Interpolated data for Ftoratur
Fig. 2: Data interpolation from different Ftorafur measurements exemplary for the amplitude (position and $\sigma$ not shown). (a): Parameter values resulting from fitting 3 Gaussian functions to the preprocessed measurement data. (b): Interpolated values used to create training data.

dependency in their amplitude (see example in Fig. 2(a)), as reported for the position of certain drug current responses [3]. Using this knowledge we can interpolate measurements from a suitable small set of calibration data. Amplitudes and positions $\left(\gamma_{n}\right)$ of Gaussian components are fit with a linear model

$$
\gamma_{n}=f\left(c, \vec{w}_{n}\right)=w_{n 0}+w_{n 1} c,
$$

with intercept $w_{n 0}$, slope $w_{n 1}$ for concentration $c$. In order to account for variability in the measurements we assume normally distributed deviations from this linear model, with variance $\beta_{n}^{-1}$ taken with respect to the calibration data set (see Fig. 2)

$$
p\left(\gamma_{n} \mid c, \vec{w}_{n}, \beta_{n}\right)=\mathcal{N}\left(\gamma_{n} \mid f\left(c, \vec{w}_{n}\right), \beta_{n}^{-1}\right),
$$

where $\mathcal{N}$ is the normal distribution. For the width of the Gaussian components we assumed normally distributed deviations around a constant mean. In order to account for the fact that measurement data contains noise, we added white noise to the training data, using random values drawn from a normal distribution. The standard deviation was estimated from the calibration data after subtracting the fit $I_{k}(V)$ from the measurements. This is still a very simplified approach and possible improvements are mentioned in the discussion section of this paper.

\section{Comparison of interpolated data}

We used Matlab ${ }^{\circledR}$ Neural Network Toolbox to simulate and train a multi layer Perceptron (MLP) with a hidden layer, consisting of 10 units. The network was trained via backpropagation using gradient descent with adaptive learning rate (Matlab ${ }^{\circledR}$ training function traingda). The predictions made by the neural network depend largely on the parameter values of the linear model used to generate the training data and consequently on the quality of the calibration data set. Based on a set of real data on several drugs (Naproxen, Flurbiprofen, Ibuprofen, Cyclophosphamide and Ifosfamide), we created a calibration data set which included all measurements for interpolating the Gaussian components. With that we generated multiple simulated data sets for training and testing the neural network. We used the leave-one-out cross validation technique to quantify the accuracy of the simulated data. For the Naproxen example, we have a total of six simulated test sets, each with only 5 measured concentrations and a different concentration excluded for data interpolation. To assess reliability of the generated data sets we calculated the Pearson correlation coefficient $R$ for each drug concentration and compared the correlation of the simulated new test sets to 


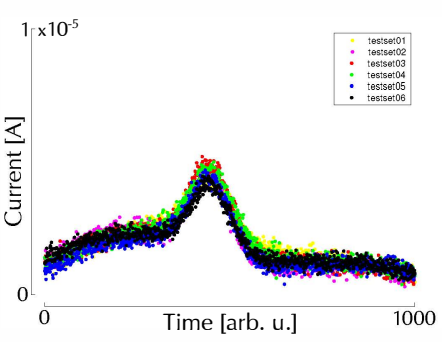

(a) Naproxen $30 \mu M$, $\mathrm{R}=0.94$

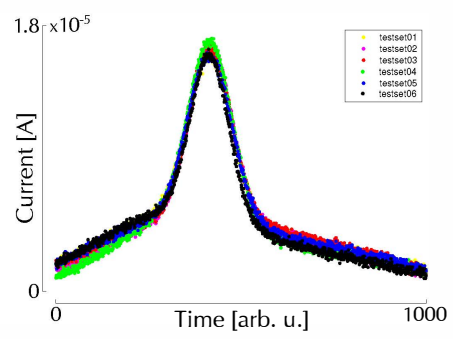

(b) Naproxen $430 \mu M, \mathrm{R}=0.99$
Fig. 3: Example for the correlation; Showing simulated data sets for Naproxen at concentrations of (a) $30 \mu M$ and (b) $430 \mu M$. For the $i$-th test-set we left the $i$-th measurement out. The time depends on the temporal resolution of the applied voltage sweep in the original measurements.

the training set that included all measurements for simulating the data. Fig. 3 shows the correlation of simulated data for Naproxen at concentrations of $30 \mu M$ and $430 \mu M$. This was repeated for every concentration and all new sets. The mean value for $R$, all test-sets and all drugs shown in Fig. 4 is $\bar{R}=0.97$ which states a high correlation of new test set and the full training set, despite the few measurement samples used. However there are some outliers that result from the random noise added to the Gaussian components. The good correlation supports the choice of the proposed algorithm for interpolating data. Figure 4, shows $R$ for different drugs plotted vs. the concentration, where each $R$ is the mean value of all test-sets for one drug. This correlation depends on the quality of the measurement data. Especially the sets for Naproxen are highly correlated because it was the best data available.

\section{RESULTS}

The results of training a neural network using simulated data generated from measurements of Naproxen at 6 different concentrations are shown in Fig. 5. Six simulated test sets are created, each of them based on a different 'reduced' calibration data set, in which one concentration is left out. The mean error for the estimated concentrations is $22.9 \mu \mathrm{M}$ which is $4.6 \%$ of the maximum concentration but $45 \%$ of the smallest measured concentration. In case the simulated data set is generated using the full calibration data set, the mean error decreases to $15.79 \mu M$ (about $3 \%$ of the maximum concentration). Similar results were obtained for the other drugs with a maximum concentration of $500 \mu M$, Flurbiprofen (mean error: $31.47 \mu M$ vs. $29.28 \mu M$ ) and Ibuprofen (mean error: $18.69 \mu M$ vs. $14.13 \mu M)$. As shown in the example of Naproxen (see Fig. 5), there is a narrower distribution of errors for the full set, than for the test sets. As expected, the 'reduced' calibration data sets produce a higher uncertainty in the concentration estimation, given the smaller concentration of data points for the interpolation. Nevertheless, the achieved error range of $3-6 \%$ is in a usable range taking into account that the US FDA accepts uncertainty of about $15 \%$ when dealing with biosensors for glucose (ISO 15197:2013). The main goal was not to create measurements with the best accuracy, but to find an approach for a cost efficient solution that is precise enough for therapeutic ranges. Our method seems to be valid for creating suitable data sets to train the classifier. Furthermore, the quality of the simulated data can be improved by increasing the size of the calibration data set

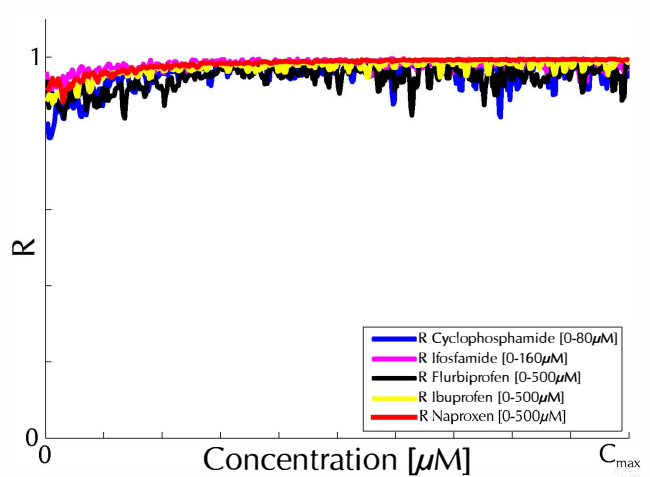

Fig. 4: Mean values of $R$ vs. concentration for several drugs. 500 concentrations per test set between 0 and $C_{\max }$. Six test sets for Naproxen, Ibuprofen and Flurbiprofen. Five for Cyclophosphamide and Ifosfamide, because there were less measurements available.

and optimizing the sensor's signal to noise ratio. However, the optimization of the sensors signal-to-noise ratio is not straightforward because the faradaic current associated with the reduction peak is dependent on the quantity of CYP immobilized onto the electrode, on the enzyme immobilization technique, and on the amount of MWCNT deposited on the electrode.

\section{MULTIPLE DRUG MEASUREMENTS WITH INTERFERENCE}

The results shown so far only deal with single drug measurements. However when multiple drugs are present the single drug current responses do not combine linearly, but are subject to non-linear interferences [7], [10]. These interferences have to be modelled in order to produce realistic simulated data for multiple drug measurements. We propose a modification of the method proposed by Carrara et al. in 2011, [7] and we incorporate the description of a single drug's current response as a sum of Gaussians as opposed to a single Gaussian

$$
i(V)=\sum_{\forall k} \Gamma_{k} C_{k}\left\{\prod_{\forall j \neq k} A_{k}\left(C_{j}\right)\right\} \frac{I_{k}(V)}{\max \left(I_{k}(V)\right)},
$$

where $I_{k}(V)$, as given by equation (1), replaces the single Gaussian term from the original equation in [7].

Since we currently lack suitable multiple-drug measurement data, we used this method to construct such data from single-drug measurements. This procedure allows us to construct a proof of concept that the proposed method can be applied to multiple drug measurements. We chose measurements of Flurbiprofen and Naproxen using CYP2C9, because both the corresponding single-drug measurement data as well as enzyme kinetics models [10] were available to us. Interference strength as well as the unknown diffusion coefficients for both drugs (PBS at $7.4 \mathrm{pH}$ ) were chosen in a way that yielded clearly recognizable, but not extreme, interference between the drugs' current responses. Figure 6 shows that a network trained on such data is able to predict concentrations with reasonable errors. The mean error for Naproxen is $52.9 \mu \mathrm{M}$ and for Flurbiprofen $55 \mu \mathrm{M}$. These results support the efficacy of this approach in the estimation of drug concentrations in multiple drug measurement data. 


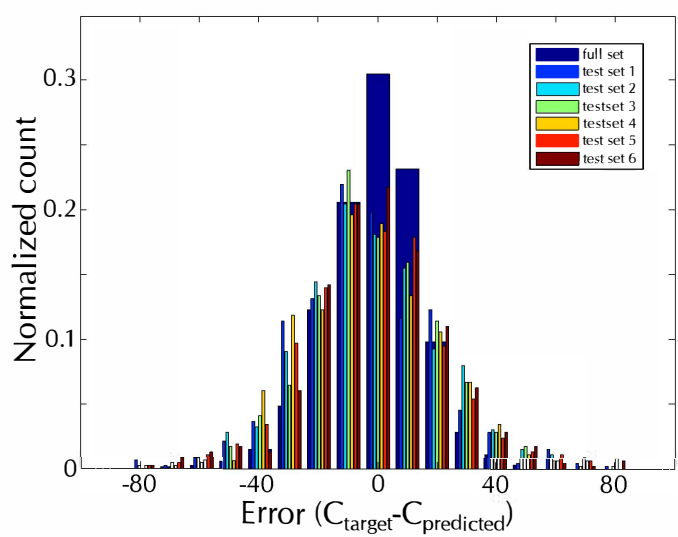

Fig. 5: Comparison of errors from a full set vs. test sets. The thick purple bars show the errors for the full set of Naproxen. The thin bars represent the test sets. Each test set excludes the i-th measurement's data for generating new data. The network was trained with the training set. Maximum measured concentration is $500 \mu M$. Random concentrations were generated between 0 and the maximum measured concentration.

\section{DISCUSSION}

We have developed a neural network approach for the estimation of drug concentrations from voltammetric measurement data. All computationally expensive steps of the proposed estimation procedure, i.e. fitting the measurement data with multiple Gaussians and training the neural network, can be delegated to a suitable computer. The results can then be used by a portable device for online drug concentration estimation at the computational cost required by simple data preprocessing and matrix operations. Since we described a novel exploratory approach to drug concentration estimation, we are confident that the current results can be improved further. The inclusion of larger calibration single-drug data sets will allow a more precise characterization of the statistics of simulated data as well as data sets suitable for the training. Multiple-drug data sets are required to confirm the validity of the simulation approach in the presence of non-linear interferences. In this work, the influence of voltage and analyte concentration on sensor noise [11] was neglected. We plan to add these effects into our model to further improve the results. The shown results represent a first milestone in the direction of the long term goal of developing portable batterypowered devices for personalized medicine. To this end, we will explore the feasibility of a spiking multi-layer Perceptron [4] implementation of the classification algorithm. This alternative approach offers the advantage of latency coding, which in turn paves the way of applying neuromorphic circuits [12] to this problem. Neuromorphic circuits make use of the subthreshold characteristics of Metal-Oxide-Semiconductor Field-Effect Transistors (MOSFETs) to build analog models of spiking neurons [13]. In the sub-threshold domain the transistor operates with very small diffusion currents that lead to a very low power consumption in the order of several $\mu W$, depending on the size of the network and the neural activity (number of spikes). Therefore a successful classification on neuromorphic spiking neural networks has high potential for supporting the development of portable devices for personalized medicine.

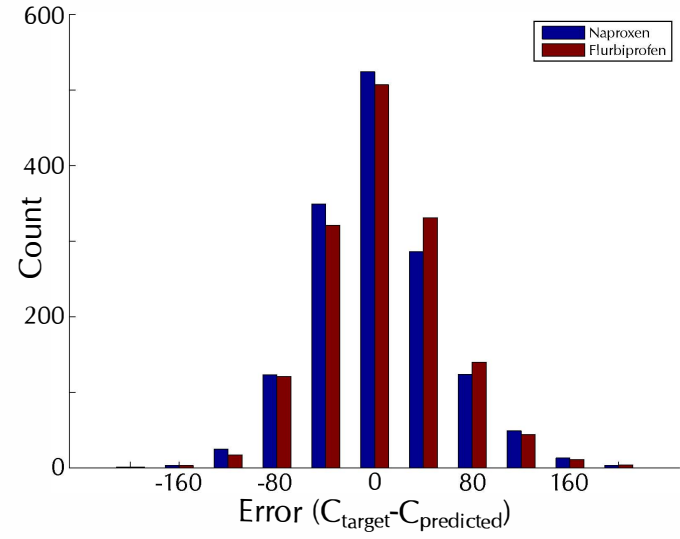

Fig. 6: Error for simultaneous prediction of Naproxen and Flurpiprofen concentration from data generated with interference. Maximum measured concentration is $500 \mu M . \epsilon_{1}: 0$, $\epsilon_{2}: 0.5$

\section{ACKNOWLEDGEMENT}

This research is supported by the DFG funded Excellence Cluster 227 (CITEC, University of Bielefeld) and the SNF Sinergia Project (code CRSII2_127547/1 and title "Innovative Enabling Micro-Nano-Bio-technologies for Implantable systems in molecular medicine and personalized therapy"). The authors would like to thank Jacob Engelmann and his research group for fruitful discussions on this topic.

\section{REFERENCES}

[1] S. Carrara, Bio/CMOS interfaces and co-design. Springer, 2012. 1, 2

[2] F. P. Guengerich, "Cytochrome p450 and chemical toxicology," Chemical research in toxicology, vol. 21, no. 1, pp. 70-83, 2007. 1

[3] C. Baj-Rossi, G. D. Micheli, and S. Carrara, "Electrochemical detection of anti-breast-cancer agents in human serum by cytochrome p450coated carbon nanotubes," Sensors, vol. 12, no. 5, pp. 6520-6537, 2012. 1,2

[4] S. M. Bohte, J. N. Kok, and H. La Poutre, "Error-backpropagation in temporally encoded networks of spiking neurons," Neurocomputing, vol. 48 , no. 1 , pp. $17-37,2002.1,4$

[5] D. Johnson, B. Lewis, D. Elliot, J. Miners, and L. Martin, "Electrochemical characterisation of the human cytochrome p450 cyp2c9," Biochemical pharmacology, vol. 69, no. 10, pp. 1533-1541, 2005. 1

[6] G. C. Baj-Rossi and S. Carrara, "A linear approach to multi-panel sensing in personalized therapy for cancer treatment," IEEE Sensor Journal, 2013. 1

[7] S. Carrara, A. Cavallini, V. Erokhin, and G. De Micheli, "Multi-panel drugs detection in human serum for personalized therapy," Biosensors and Bioelectronics, vol. 26, no. 9, pp. 3914-3919, 2011. 1, 2, 3

[8] A. Yarman, U. Wollenberger, and F. W. Scheller, "Sensors based on cytochrome p450 and cyp mimicking systems," Electrochimica Acta, vol. 110 , pp. $63-72,2013.1$

[9] A. J. Bard and L. R. Faulkner, Electrochemical methods: fundamentals and applications. Wiley New York, 1980, vol. 2. 2

[10] T. S. Tracy, "Atypical enzyme kinetics: their effect on in vitro-in vivo pharmacokinetic predictions and drug interactions," Current drug metabolism, vol. 4, no. 5, pp. 341-346, 2003. 3

[11] S. S. Ghoreishizadeh, G. Nanda, S. Carrara, and G. De Micheli, "Empirical study of noise dependence in electrochemical sensors," in Advances in Sensors and Interfaces (IWASI), 2013 5th IEEE International Workshop on. IEEE, 2013, pp. 36-39. 4

[12] E. Chicca, F. Stefanini, C. Bartolozzi, and G. Indiveri, "Neuromorphic electronic circuits for building autonomous cognitive systems," Proceedings of the IEEE, vol. 102, no. 9, pp. 1367-1388, Sept 2014. 4

[13] S.-C. Liu, J. Kramer, G. Indiveri, T. Delbruck, and R. Douglas, Analog VLSI:Circuits and Principles. MIT Press, 2002. 4 